mid-range ejection fraction (HFmrEF) Vs. Heart failure with preserved ejection fraction $(\mathrm{HFpEF})$ and the underlying cause of heart failure. $42 \%$ of patients had a daily weight charts, and weight was recorded for $67 \%$ of the cumulative days in hospital. $52 \%$ of patients had fluid balance charts, and were totaled on $29 \%$ of admission days (figure 1).

There were symptoms and signs of pulmonary oedema in $28 \%$ of patients, while $5 \%$ of patients had only signs of peripheral oedema, and $67 \%$ had symptoms and signs of both pulmonary and peripheral oedema (figure 2).

Conclusions Two thirds of patients admitted with AHF and requiring IV diuretics have reduced LV ejection fraction, ischemic heart disease is the most common underlying cause of heart failure among this population. Despite guidelines recommendations, close monitoring of weight and fluid balance in patients hospitalized due to acute heart failure, is not being documented as much as desired.

Conflict of Interest None

\section{ROLE OF THE DAY CARE UNIT IN THE OPTIMAL MANAGEMENT OF HEART FAILURE PATIENTS}

${ }^{1}$ Stuart Rosen, ${ }^{2}$ Ekanjali Dhillon, ${ }^{2}$ Harman Singh. ${ }^{1}$ National Heart and Lung Institute, Southall, UK; ${ }^{2}$ London Northwest University Healthcare NHS Trust

10.1136/heartjn--2021-BCS.145

We set up our ambulatory care unit and heart failure (HF) lounge to improve management of heart failure (HF) patients; reduce A\&E attendances and hospital admissions; optimise medicine administration; facilitate discharge from our wards and promote an holistic approach. We kept the lounge running as a 'green' zone through the Covid pandemic in 2020.

Methods The programme used a combination of quality improvement and Lean methodology working with staff and patients to create new pathways The service processes were reviewed and referral pathways aligned to support community to specialist referral and redesign of service was completed. The team monitored quality outcomes such as patients access to specialist review, medication review and patient experience of the service, also identifying patterns of attendance and admission pre and post attendance to the lounge.

Results The evaluation of the programme, centred on the experience of staff and patients, has shown improved information sharing between primary and secondary care staff; the patients described the care as efficient, enabling them to remain in their own home, with effective continuity of care. In 2020, despite the pandemic, the ACU was kept as a 'green' zone and 521 patients attended; - 87\% of patients referred from community services did not have to attend A\&E; - 100\% of patients received a Specialist Review; $100 \%$ of patients received a full medication review and optimisation; - there was a reduction in A\&E attendance following attendance to the lounge of $90 \%$. - There was a reduction in bed days of 310 , with a cost reduction of $£ 231,649$.

Conclusion The ACU Heart lounge has proven that the day case concept can be extremely useful in managing complex chronic disease like heart failure, benefiting patients and service providers, even in circumstances of the continuing Covid19 pandemic.

Conflict of Interest None

\section{IMPACT OF COVID-19 PANDEMIC ON GLOBAL CONTRIBUTIONS TO THE EUROPEAN HEART FAILURE CONGRESS: DO WE NEED MORE DIVERSITY?}

Zainab Dakhil. IBN Al Bitar Cardiac Centre, Al-Kindy College of Medicine/University of Baghdad, Baghdad, Iraq

\subsection{6/heartjnl-2021-BCS.146}

Background Highly impact international conferences play a critical role in advancing career, academic thrive and global networking. After COVID-19 era, virtual science contributes remarkably to increase networking and professional collaboration across the globe. Up to our knowledge there is no data regarding global contributions to cardiology conferences in general and how COVID-19 might impact the landscape of these contributions in particular, especially in the context of contributions from low- and middle-income countries (LIC and MIC).

Purpose Current analysis aimed to determine the global diversity in abstracts presented at European Heart Failure congress according to the continent and world bank class of the country of the first author.

Methods Cross sectional analysis of the European Heart Failure congress abstracts published in the (European Journal of Heart Failure) in 2019 and 2020 which represented the before and during COVID-19 era respectively. The analysis was done according to the continent and world economic classification of the first authors' country.

Results Total n. of abstracts analysed were 2779 with $17.6 \%$ decline rate during pandemic contributions (1634 in 2019 vs 1145 in 2020). Europe followed by Asia had the highest total abstract contributions; 1701 and 740 abstracts respectively. Europe contributions proportion increased during pandemic (60.52\% in 2019 to $62.18 \%$ in 2020) while Asia contributions proportion declined during pandemic $(28.33 \%$ in 2019 vs $24.19 \%$ in 2020), $\mathrm{p}=0.03$, see figure 1 . Russia had the highest number of contributions (11.68\% in 2019 vs $12.22 \%$ in 2020), this is followed by Spain $(7.34 \%$ in 2019 vs $11.35 \%$ in 2020) and Portugal (5.87\% in 2019 vs $11.44 \%$ in 2020). A total of $1899(68.33 \%)$ abstracts were from high-income countries (HIC) vs 880 (31.67\%) from MIC and none from LIC in the sum of both years. Contributions from MIC increased during COVID-19 era from 30.23\% in 2019 to $33.71 \%$ in 2020 , while contributions from HIC declined from $69.76 \%$ in 2019 to $66.28 \%$ during COVID-19 era in 2020 , $\mathrm{p}=0.052$, see figure 1 .

Conclusions About third of contributions to European Heart Failure congress were from MIC and none from LIC, however, there is modest increase of contributions from MIC

Global Distribution of Abstracts Presented in HFA Before and During COVID-19 According to First Authors' Continent

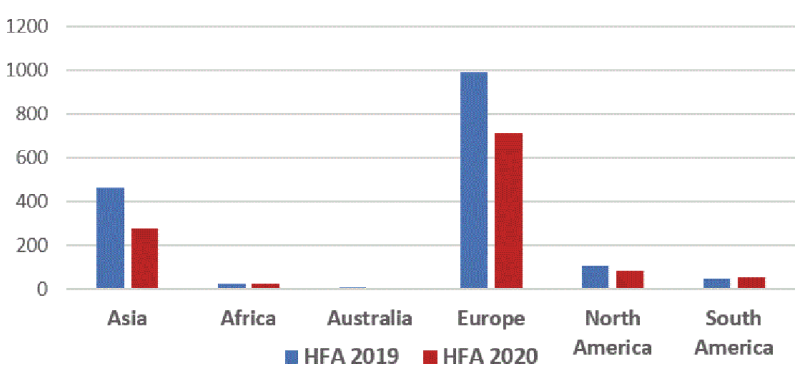

Abstract 149 Figure 1 
Global Distribution of Abstracts Presented in HFA Before and During COVID-19 According to First Authors' Country

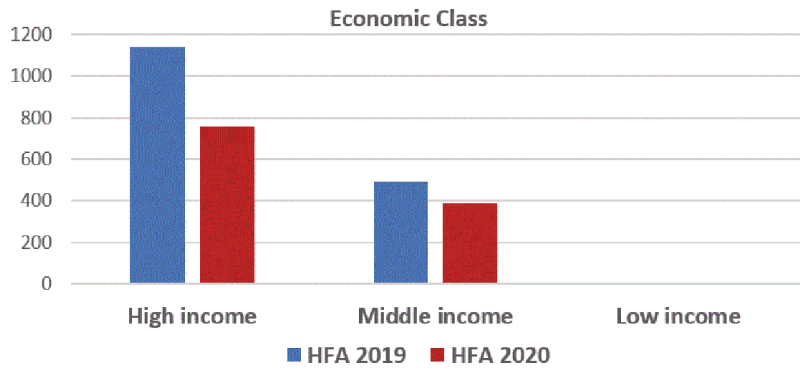

Abstract 149 Figure 2

during COVID-19 era which can be explained by more presentations from these countries due to feasible virtual meetings and waiving of conference fees in 2020 (HFA Discoveries Platform). Further networking and collaborations with researchers from these countries should be encouraged especially with the high cardiovascular disease burden in these countries which necessitated sharing their perspectives and experiences to decrease cardiovascular disease burden worldwide especially by using the hybrid approach and virtual science. Increasing research, travel and educational grants (especially for researchers from LIC) along with calling for action to remove other barriers like VISA issues can help to bridge the conference equity gap and achieve solidarity in science.

Conflict of Interest None

\section{SGLT2 INHIBITION IN HEART FAILURE WITH A REDUCED EJECTION FRACTION: HOW MANY PATIENTS WOULD BENEFIT?}

Jacquelyn Hooper, Steven Shaw, Paul Callan, Simon Williams. Wythenshawe Hospital, Manchester, Manchester, UK

\subsection{6/heartjnl-2021-BCS.147}

Introduction Sodium-glucose co-transporter (SGLT2) inhibition has been shown to reduce the risk of cardiovascular death or hospitalisation in patients with heart failure with reduced ejection fraction (HFrEF) with or without type 2 diabetes mellitus (T2DM). Since publication of the DAPA- $\mathrm{HF}^{1}$ and EMPEROR$\mathrm{HF}^{2}$ trials and UK NICE approval, ${ }^{3}$ SGLT2 inhibitor prescription has been increasing for patients with HFrEF.

Methods Electronic medical records were examined for all patients reviewed in heart failure clinic (including virtual consultation) at Wythenshawe hospital, Manchester over a 4 week period (25/01/21 - 19/02/21). Data obtained was stratified according to NYHA class, LV ejection fraction (LVEF) and presence or absence of T2DM.

Results 232 patients with a diagnosis of heart failure had either face to face or virtual telephone review over the specified time period. Patient baseline characteristics were comparable to patients recruited into the DAPA-HF and EMPEROR-HF trials*. Mean age 67 years (ranging from 20-94 years). $68 \%$ were male. $25 \%$ had T2DM. $19 \%$ $(n=45)$ were subsequently excluded from further analysis due to improvement in LVEF $>40 \%$ (mean time since last echo 5.4 months) or heart failure with preserved ejection fraction, along with 84 patients who were asymptomatic
(NYHA I). 122 patients met the eligibility criteria* for SGLT2 inhibition: of whom 93\% were already established on standard HF treatment (the combination of a betablocker, an aldosternone antagonist or either an ACE inhibitor, or angiotensin II receptor blocker \pm neprolysin inhibitor. $8 \%$ were continuing uptitration of their standard heart failure treatments. In those not on standard HF therapy, a contraindication for the absent therapy was recorded in $50 \%$ of cases. $24 \%(n=29)$ were already prescribed SGLT2 inhibitors. Of the $30 \%(n=37)$ of patients who had T2DM, $43 \%(n=16)$ were prescribed SGLT2 inhibitors, leaving over half without. Amongst the non-diabetic population of eligible patients $(n=86)$, only $15 \%$ were taking SGLT2 inhibitors. Eligible patients taking SGLT2 inhibitors $(n=29)$ were prescribed Dapagliflozin in $79.3 \%$ of cases, with Empagliflozin and Canagliflozin prescribed much less commonly $(10.3 \%$ and $10.3 \%$ respectively). Of those in the SGLT2 cohort, Dapagliflozin was commenced at the most recent clinic appointment in $45 \%$ of cases indicating a rapid uptake in the prescription of these agents.

Conclusion In line with recent NICE guidance, the use of SGLT2 inhibition in the HFrEF population is beginning to increase. There is scope for optimisation in treatment. In our cohort of an unselected group of consecutive patients In heart failure, 122/232 (53\%) patients were eligible for the treatment. Of the patients with T2DM, 57\% were not yet treated with SGLT2 inhibitors. The proportion of nondiabetic eligible patients not treated with SGLT2 inhibition was predictably higher (85\%). However, this figure is likely to fall significantly over the next year as awareness of this new treatment increases and local guidance includes this class of agent.Eligibility criteria* for SGLT2 inhibition included: LV ejection fraction $<40 \%$, symptomatic heart failure (NYHA II or above) and an eGFR $>30$. Those with type 1 diabetes and a history of recurrent UTI were not deemed as eligible.

Conflict of Interest No

\section{REFERENCES}

1. McMurray JJV, Solomon SD, Inzucchi SE, Kober L, Kosiborod MN, Martinez FA Ponikowski P, Sabatine MS, Anand IS, Belohlavek J, et al. Dapagliflozin in patients with heart failure and reduced ejection fraction. $N$ Engl J Med 2019;381:1995-2008. doi: 10.1056/NEJMoa1911303

2. Packer M, Anker SD, Butler J, Filippatos G, Pocock SJ, Carson P, Januzzi J, Verma $\mathrm{S}$, Tsutsui H, Brueckmann M, et al; EMPEROR-Reduced Trial Investigators. Cardiovascular and renal outcomes with empagliflozin in heart failure. $N$ Engl J Med 2020;383:1413-1424. doi: 10.1056/NEJMoa2022190

3. National Institute for Health and Care Excellence NICE technology appraisal guidance TA679.

\section{THE PRE-HFPEF ENTITY: A WINDOW OF OPPORTUNITY TO PREVENT AND HALT THE PROGRESSION TO HF WITH PRESERVED EJECTION FRACTION (HFPEF)}

Patrick Tran, Ross Obukofe, William Thompson, Nimai Desai, Nayaab Abdul Kader, Prithwish Banerjee. University Hospitals Coventry and Warwickshire, Coventry, UK

\subsection{6/heartjnl-2021-BCS.148}

Background Before the emergence of HFpEF, there is an insidious phase of progressive myocardial fibrosis, arterial stiffness, and rising left ventricular end-diastolic pressures (LVEDP) driven by amassing cardio-metabolic comorbidities. This phase is characterized by incipient structural cardiac 